\section{Primary Aldosteronism Simulating Polymyositis}

\section{To the Editor:}

Primary aldosteronism (PA) is a condition in which tumors or hyperplasia of the zona glomerulosa of adrenal cortex cause overproduction of aldosterone and suppression of renin activity, resulting in refractory hypertension, hypokalemic paralysis, and potassium-losing nephropathy. Most patients present with typical features and are easily diagnosed. However, some patients may have special presentations and represent a major challenge for diagnosis and treatment. For example, patients with PA prominently characterized by hypokalemic myopathy (HM) may resemble patients with polymyositis (PM) clinically and pathologically.

We describe 3 cases of PA who presented to our hospital with HM as a prominent feature of their hyperaldosteronism. Findings in these patients included muscle pain and weakness, significantly increased creatine kinase (CK), myopathic changes on electromyography (EMG), or inflammatory cell infiltration on muscle biopsy. Early diagnosis and treatment for these patients were delayed because of the PM-like findings. The clinical and enzymatic features of muscle involvement and the electromyographic and/or histological findings of muscles were substantially consistent with the diagnosis of $\mathrm{PM}^{1}$

Few cases of PA chiefly characterized by HM have been reported. A search of reports published in China over the past decade identified 7 cases ${ }^{2,3,4,5,6,7}$. In order to increase clinicians' awareness and understanding of PA simulating PM and prevent possible misdiagnosis, we analyzed 3 cases admitted to our institution and 7 treated by others.

Case 1. A 45-year-old previously healthy man presented to our outpatient department with muscle myalgia and weakness involving all extremities for 10 days. Serum CK was 18,561 IU/l and EMG confirmed myogenic damage. He was admitted for suspected PM. Blood pressure (BP) was 185/115 mm Hg. He had a passive position. No rash was observed and thyroid gland was not enlarged. Examination of heart, lung, and abdomen revealed no abnormalities. Muscle power was grade 2/5 over proximal and distal muscle groups in all 4 limbs. Sensory testing was normal. Biceps and patellar reflexes were diminished and plantar response was downward. Laboratory tests revealed the following values: serum potassium 1.24 $\mathrm{mmol} / \mathrm{l}$, sodium $140.9 \mathrm{mmol} / \mathrm{l}$, chloride $101.2 \mathrm{mmol} / \mathrm{l}$, magnesium 0.62 $\mathrm{mmol} / \mathrm{l}$, calcium $2.11 \mathrm{mmol} / 1$, carbon dioxide $41.8 \mathrm{mmol} / \mathrm{l}$; myocardial enzyme profile: creatine phosphokinase $18,561 \mathrm{IU} / 1$, CK isoenzyme (CK-MB) $82.6 \mathrm{IU} / 1$, alanine aminotransferase (ALT) $385 \mathrm{IU} / 1$, aspartate aminotransferase (AST) 825 IU/1, lactate dehydrogenase (LDH) 1158 IU/l; urine dip test: blood +++, protein ++, PH 8.0, and 3 red blood cells (RBC) per high power field (hpf). Complete blood count, erythrocyte sedimentation rate (ESR), blood urea nitrogen (BUN), creatinine, glucose, total protein, albumin, and thyroid function tests were normal. Normal immunologic measures included antinuclear antibody (ANA), antiextractable nuclear antigen (ENA) antibodies, anti-double stranded DNA (dsDNA) antibodies, complement, immunoglobulins, and rheumatoid factor (RF). Electrocardiography (ECG) showed sinus rhythm, flat $\mathrm{T}$ waves in all leads, obvious $\mathrm{U}$ waves, fusion of $\mathrm{T}$ and $\mathrm{U}$ waves, and ST-segment depression of $0.10-0.15 \mathrm{mV}$ in V3-6. Chest radiographs were normal. B-mode ultrasonography of bilateral kidneys detected no abnormalities. Quadriceps muscle biopsy showed focal necrosis of muscle fibers with inflammatory cell infiltration. Vacuolar changes in muscle fibers were not seen (Figure 1). During hospitalization, he received intravenous methylprednisolone $120 \mathrm{mg} /$ day, aggressive antihypertensive therapy, and potassium supplementation $(6 \mathrm{~g} /$ day potassium chloride). After 5 days of treatment, his myalgia and muscle weakness improved significantly and testing revealed CK $762 \mathrm{IU} / \mathrm{l}$ and potassium $2.9 \mathrm{mmol} / \mathrm{l}$. This treatment course was not consistent with PM. Considering the presence of concomitant hypertension, hypokalemia, and alkalemia, we agreed that he was more likely to have PA than PM. Steroid use was then discontinued. Further investigations revealed 24-h urinary potassium excretion $69.5 \mathrm{mmol} / \mathrm{l}$, plasma aldosterone $554.2 \mathrm{pmol} / \mathrm{l}$ in supine position (normal 166.2-470.9), and plasma rennin activity $0.04 \mu \mathrm{g} / 1 / \mathrm{h}$ in supine position (normal $0.2-1.9$ ). Contrast-
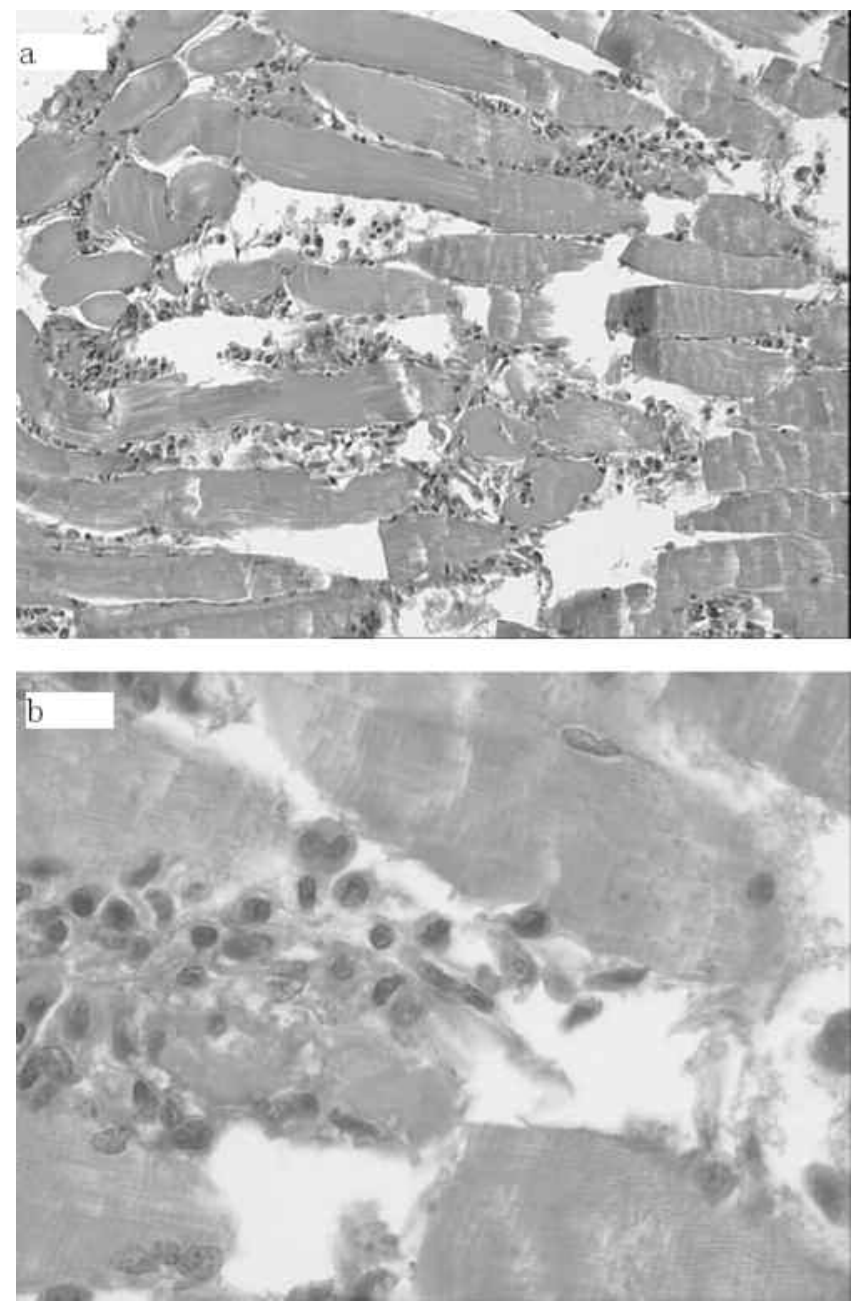

Figure 1. Biopsy specimen of striated muscle shows destruction of muscle fascicles and focal necrosis of muscle fibers, with infiltration of many histiocytes, lymphocytes, and neutrophils between striated muscle fascicles; and no vacuolar changes in the muscle fibers (a: H\&E, original magnification $\times 100$; b: H\&E, original magnification $\times 400$ ).

enhanced computed tomography (CT) scan of bilateral adrenal glands showed a $1.1 \mathrm{~cm} \times 1.2 \mathrm{~cm}$ adenoma in the left adrenal area. After surgical removal of the adenoma, the pathology report confirmed adrenocortical adenoma. Postoperative tests showed potassium $4.5 \mathrm{mmol} / 1$ and CK 65 IU/l. BP was normal and the myalgia and muscle weakness resolved. The patient reported no recurrence of the symptoms during 2-year followup.

Case 2. A 36-year-old woman visited the outpatient department due to 1 week of worsening muscle pain and weakness in all extremities, which had occurred repeatedly during the past 3 months. Evaluation revealed CK $6954 \mathrm{IU} / 1$ and EMG showed myogenic damage. She then was hospitalized for suspected PM. She admitted a history of polydipsia and polyuria for the last 6 months and had never had BP measured. BP was 165/105 mm Hg. She had a staggering gait. No rash was observed and the thyroid gland was not enlarged. Examination of heart, lung, and abdomen revealed no abnormalities. Her power was grade $3 / 5$ over the proximal muscle groups and grade $4 / 5$ for distal muscle groups in all 4 limbs. Muscle tenderness was detected in all 4 limbs. Sensory testing was normal. Knee reflex was diminished and plantar response was downward. Laboratory tests showed potassium $1.85 \mathrm{mmol} / 1$, sodium $145.3 \mathrm{mmol} / 1$, chloride $99.4 \mathrm{mmol} / 1$, magnesium $0.72 \mathrm{mmol} / \mathrm{l}$, calcium $2.25 \mathrm{mmol} / 1$, and $\mathrm{CO}_{2} 34.8 \mathrm{mmol} / \mathrm{l}$; myocardial enzyme profile: CK 6954 IU/1, CK isoenzyme 45 IU/1, ALT 255 IU/1, AST 
687 IU/1, and LDH 948 IU/1; urine dip test: blood (-), protein (-), and PH 7.0. No abnormalities were found for complete blood count, ESR, BUN, creatinine, glucose, total protein, albumin, and thyroid function tests. Immunologic tests were normal including ANA, anti-ENA, anti-dsDNA, complement, immunoglobulins, and RF. ECG showed sinus rhythm and ST-segment depression. Thoracic radiography was also normal. B-mode ultrasonography of bilateral kidneys detected no abnormalities. Her myalgia and muscle weakness improved completely 1 week after aggressive BP lowering therapy and potassium supplementation. Repeated tests revealed CK $138 \mathrm{IU} / 1$ and potassium $3.1 \mathrm{mmol} / 1$. She was considered to be more likely to have PA. Further investigations revealed 24-h urinary potassium excretion $57.69 \mathrm{mmol} / \mathrm{l}$, plasma aldosterone concentration $495.3 \mathrm{pmol} / \mathrm{l}$ in supine position, and plasma rennin activity $0.05 \mu \mathrm{g} / \mathrm{l} / \mathrm{h}$ in supine position. CT scan of bilateral adrenal glands detected a $1.0 \mathrm{~cm} \times 1.1 \mathrm{~cm}$ adenoma of the right adrenal gland that was confirmed in the pathology report after surgical resection. Postoperative tests showed potassium $4.6 \mathrm{mmol} / \mathrm{l}$ and $\mathrm{CK}$ $61 \mathrm{IU} / 1$. BP was within normal range, and muscle power was grade $5 / 5$ in all 4 limbs. None of the symptoms recurred during 1 year followup.

Case 3. A 41-year-old woman presented to the outpatient department with muscle weakness involving all extremities for 1 week and muscle myalgia for 5 days. Examination showed CK $3565 \mathrm{IU} / 1$ and EMG detected no abnormalities. She then was admitted for suspected PM. She reported a history of hypertension for 1 year (highest, 185/110 mm Hg). Current BP was 180/108 mm Hg. She assumed an active position. No rash was observed and thyroid gland was not enlarged. Examination of heart, lung, and abdomen revealed no abnormalities. Muscle power was grade $4 / 5$ over the proximal muscle groups and 5/5 over the distal muscle groups in all 4 limbs. Sensory testing was normal. Patellar reflex was weakened and plantar response was downward. Laboratory tests: potassium $2.0 \mathrm{mmol} / 1$, sodium $146.8 \mathrm{mmol} / 1$, chloride $100.1 \mathrm{mmol} / \mathrm{l}$, magnesium $0.95 \mathrm{mmol} / \mathrm{l}$, calcium $2.32 \mathrm{mmol} / 1$, and $\mathrm{CO}_{2} 32.5 \mathrm{mmol} / \mathrm{l}$; myocardial enzyme profile: $\mathrm{CK}$ $3565 \mathrm{IU} / 1, \mathrm{CK}$ isoenzyme $33 \mathrm{IU} / 1$, ALT $95 \mathrm{IU} / 1$, AST $109 \mathrm{IU} / 1$, and LDH 335 IU/1; urine dip test: blood (-), protein (-), PH 7.0. Complete blood count, ESR, BUN, creatinine, glucose, total protein, albumin, and thyroid function were normal. Immunologic tests were normal. ECG showed sinus rhythm and ST-segment depression in all leads, with flat $\mathrm{T}$ waves and visible $\mathrm{U}$ waves. Chest radiographs were normal. B-mode ultrasonography of bilateral kidneys showed a $1.5 \mathrm{~cm}$ well defined hypoechoic mass in the left adrenal area. Her myalgia and muscle weakness resolved 5 days after aggressive BP lowering therapy and potassium supplementation. Repeated tests revealed CK $152 \mathrm{IU} / \mathrm{l}$ and potassium $2.9 \mathrm{mmol} / \mathrm{l}$. Further investigations revealed 24-h urinary potassium excretion $49.55 \mathrm{mmol} / \mathrm{l}$, plasma aldosterone $510.8 \mathrm{pmol} / \mathrm{l}$ in supine position, and plasma rennin activity $0.12 \mu \mathrm{g} / \mathrm{l} / \mathrm{h}$ in supine position. Adrenal CT scan showed a left adrenal adenoma of $1.1 \mathrm{~cm} \times 2.0 \mathrm{~cm}$ size, confirmed by the pathology report after surgical removal. Postoperative evaluations revealed potassium $4.1 \mathrm{mmol} / 1$ and CK 44 IU/1; BP remained in the normal range. She did not develop any further symptoms during followup of 6 months.

This case series consists of 3 men and 7 women aged 27-51 years (mean 39 yrs) who presented with myalgia, muscle weakness, and increased muscle enzymes as prominent symptoms. In 2 of the 10 patients, these symptoms were initial manifestations. Five patients reported a history of hypertension for 6 months to 8 years prior to presentation; 5 had a history of polydipsia and polyuria for 6 months to 3 years; and 1 described repeated edema of the face and lower extremities (alleviated after rest) 2 years prior to presentation. Nine patients were suspected of having PM by outpatient care providers and 1 was diagnosed with suspected acute non-Q wave myocardial infarction. During hospitalization, 2 patients received high doses of steroids (80-120 mg/day methylprednisolone) for suspected PM as evidenced by myalgia, muscle weakness, extremely high CK level, and electromyographically detected myogenic damage (Table 1).

Eight patients with PA experienced significant myalgia and muscle weakness and 2 had only muscle weakness. Muscle myalgia was found in all 4 extremities in 7 patients and in the lower extremities in 1 patient. Muscle weakness was present in the extremities in 9 patients and in the lower extremities in 1 . Limb power was grade $2 / 5$ to $5 / 5$ in all patients. Three patients had muscle tenderness involving all limbs and 1 reported numbness of the ends of the limbs. Eight patients had diminished deep tendon reflexes. Sensory systems and cranial nerve examinations were normal in all 10 patients, with downward plantar response. All 10 patients had high BP (140-210/90-130 mm Hg) on admission (Table 1).

Auxiliary examinations included laboratory test results: Significantly increased CK (up to the highest level of 18,561 IU/l) was found in all 10 patients. No increase was found or only a slight increase was noted in 8 patients who underwent CK isoenzyme tests. All 10 patients (100\%) had elevated LDH; 9/9 patients tested had increased AST and 5/5 tested had increased ALT. Significantly low serum potassium (lowest, $1.24 \mathrm{mmol} / \mathrm{l}$ ) was found in all 10 patients. Nine of 9 patients tested had increased 24-h urinary potassium excretion; 7/7 patients tested had increased $\mathrm{CO}_{2}$. Among 2 patients who underwent blood gas analysis, 1 had increased $\mathrm{pH}$ and 1 had normal $\mathrm{pH}$ values. Measurements of serum sodium in 7 patients revealed normal or slightly elevated levels $(140.7-151 \mathrm{mmol} / \mathrm{l})$, and serum chloride in 6 patients showed normal or mildly low levels $(95.1-101.6 \mathrm{mmol} / \mathrm{l})$. In 4 patients who had urinalysis, $\mathrm{pH}$ was neutral or alkaline in the range of 7.0-8.0. In 1 of the 4 patients, urine dip test showed $2+$ protein and $3+$ blood, but microscopy revealed only $3 \mathrm{RBC} / \mathrm{hpf}$, suggesting myoglobinuria. Seven of 8 patients $(87.5 \%)$ had increased plasma aldosterone concentration. Five of 5 patients tested had decreased supine plasma rennin activity. Evaluations of blood cortisol in 4 patients showed normal levels. ESR and immunologic tests (ANA, anti-ENA, anti-dsDNA, complement, immunoglobulins, and RF) in 4 patients found all measures were normal. In the 3 patients treated in our institution and 2 reported elsewhere who had complete data, complete blood counts, ESR, BUN, creatinine, uric acid, glucose, total protein, albumin, magnesium, calcium, and thyroid function tests were normal (Table 1). ECG showed hypokalemic changes in 9 patients and normal findings in 1 . Among 9 patients undergoing EMG, 5 had myogenic damage and 4 showed no evidence of abnormalities (Table 1).

Adrenal B ultrasound examination in 8 patients showed no abnormal findings in 6, but a unilateral well defined hypoechoic mass in 2 patients. Unenhanced or contrast-enhanced CT scans of adrenal glands in 9 patients showed adrenal tumor or mass on the left $(n=5)$ or the right side $(n=4)$. In 1 patient who underwent adrenal magnetic resonance imaging, an adrenal tumor was detected on the right side. Among 9 patients whose adenoma or mass was surgically removed, pathology reports confirmed adrenocortical adenoma in 8 and adrenocortical hyperplasia in 1. In 3 patients who underwent muscle biopsy, degeneration/necrosis of muscle fibers and inflammatory cell infiltration were present, with the absence of vacuolar changes (Table 1).

Potassium supplementation, antihypertensive therapy, and surgical resection of adrenal adenoma (ablative therapy was performed in 1 patient) resolved myalgia and muscle weakness and restored blood potassium, muscle enzyme levels, and BP to normal in all patients. In 8 patients followed up for 6 months to 5 years, no recurrences were reported.

PA is an endocrine disorder with 2 main subtypes: aldosterone-producing tumors (adenomas) and idiopathic hyperaldosteronism. Patients with PA may present with hypertension, neuromuscular dysfunction, potassiumlosing nephropathy, or hypokalemia. Hypertension is the first and most common symptom. In special cases, severe skeletal muscle injury may be complicated and hypokalemic myopathy (HM) may occur. PM refers to nonsuppurative inflammation of striated muscle due to an autoimmune cause, which is characterized by symmetric proximal muscle weakness, myalgia, elevated serum muscle enzymes, myogenic damage on EMG, and varying degrees of muscle inflammation and destruction confirmed pathologically. Glucocorticoids are considered the first choice for treatment, and are usually used in combination with immunosuppressive agents. Glucocorticoids may decrease muscle enzymes to almost normal levels and progressively improve muscle strength within 6-12 weeks after start of treatment. When muscle enzymes return to normal levels, the dose of glucocorticoids should be decreased and maintained at a lower level for continued use for more than 2 years, since inflammatory myopathy requires 
Table 1. Clinical information and treatment of 10 cases of primary aldosteronism (PA) prominently characterized by hypokalemic myopathy (HM); 3 from our hospital and 7 reported by others.

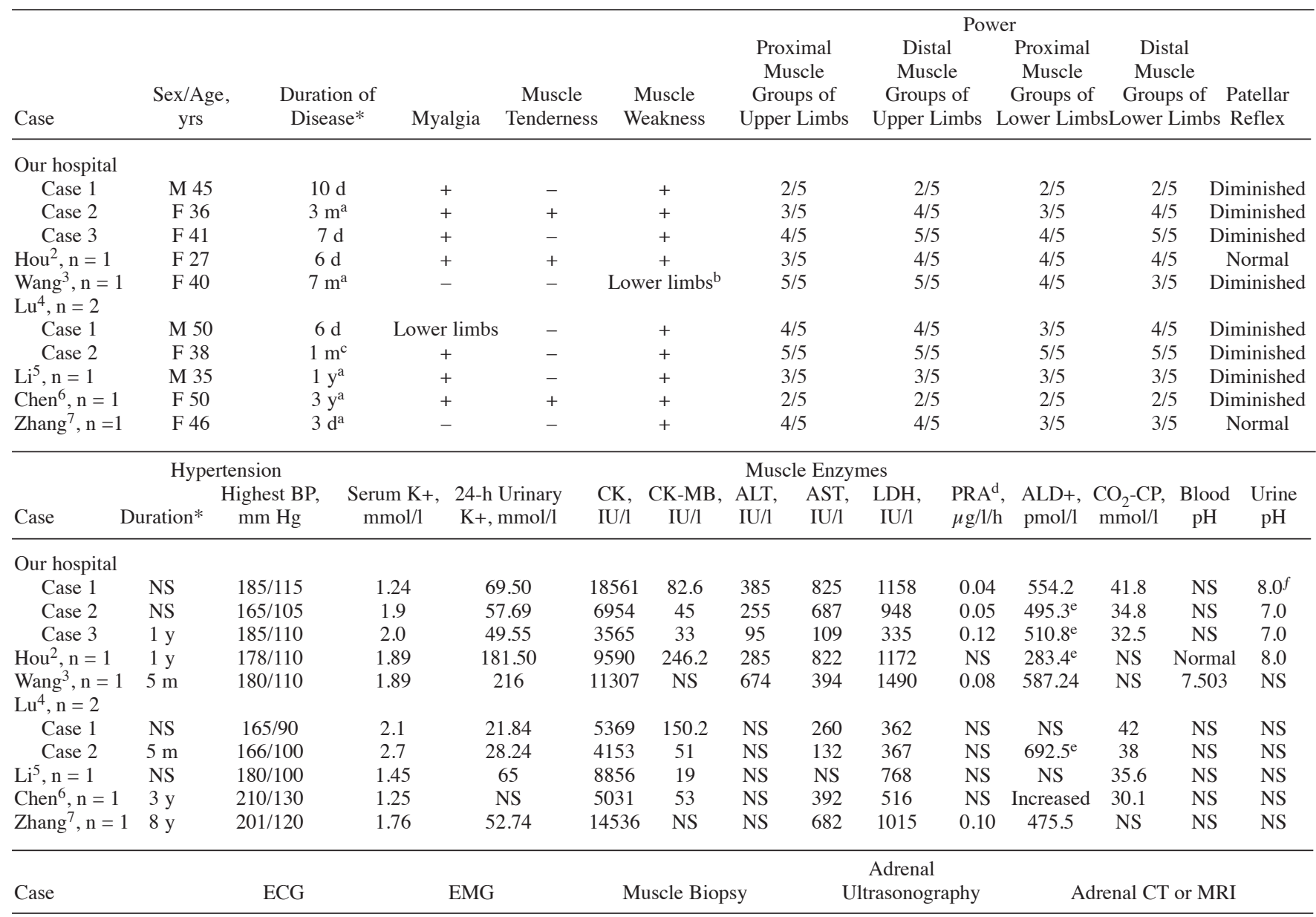

Our hospital

Case 1

Flat $\mathrm{T}$ waves, obvious U waves, Myo ST segment depression

Case 2

Case 3

ST segment depression

Flat $\mathrm{T}$ waves, visible $\mathrm{U}$ waves, ST segment depression

$\mathrm{Hou}^{2}, \mathrm{n}=1 \quad$ No abnormality $\quad$ Myo

Wang $^{3}, \mathrm{n}=1 \quad$ Flat $\mathrm{T}$ waves, obvious $\mathrm{U}$ waves, No ST segment depression

$\mathrm{Lu}^{4}, \mathrm{n}=2$

Case 1 Flat and inverted $\mathrm{T}$ waves, minor $\mathrm{U}$ waves, ST segment depression

Case 2 ST depression

Myo

No

$\mathrm{Li}^{5}, \mathrm{n}=1 \quad$ Flat $\mathrm{T}$ waves, obvious $\mathrm{U}$ waves, Myo ST segment depression

$\mathrm{Chen}^{6}, \mathrm{n}=1 \quad$ Flat $\mathrm{T}$ waves, obvious $\mathrm{U}$ waves, Not done ST segment depression

Zhang $^{7}, \mathrm{n}=1 \quad$ Left ventricular hypertrophy, prolonged Q-T interval
Focal degeneration and necrosis of muscle fibers, inflammatory cell infiltration, with absence of vacuolar changes

Not done

Not done

Partial degeneration, necrosis and regeneration of muscle fibers, and macrophage response, interstitial edema, a small number of lymphocytes, mononuclear cell infiltration

Focal degeneration, necrosis and disintegration of muscle fibers, mononuclear cell infiltration, with absence of muscle regeneration

Not done

Not done

Not done

Not done

Not done
No

Left adrenal adenoma $1.1 \mathrm{~cm} \times 1.2 \mathrm{~cm}$

No Right adrenal adenoma $1.0 \mathrm{~cm} \times 1.1 \mathrm{~cm}$ Left adrenal Right adrenal adenoma $1.1 \mathrm{~cm} \times 2.0 \mathrm{~cm}$ hypoechoic mass $1.5 \mathrm{~cm}$ Right adrenal hypoechoic mass $1.4 \mathrm{~cm}$

Right adrenal mass $1 \mathrm{~cm} \times 2 \mathrm{~cm}$

Not done

Left adrenal nodule $0.8 \mathrm{~cm} \times 0.8 \mathrm{~cm}$

No

Left adrenal adenoma $1.1 \mathrm{~cm} \times 1.2 \mathrm{~cm}$

No

Left adrenal adenoma $1.1 \mathrm{~cm} \times 1.2 \mathrm{~cm}$

No Right adrenal mass $1.5 \mathrm{~cm} \times 2.5 \mathrm{~cm}$

Not done

Right adrenal adenoma $2.5 \mathrm{~cm} \times 2.5 \mathrm{~cm}$, mild hyperplasia of left adrenal gland Short T1 and long T2 in right adrenal area $1.34 \mathrm{~cm} \times 1.40 \mathrm{~cm} \times 1.94 \mathrm{~cm}$; MRI findings 


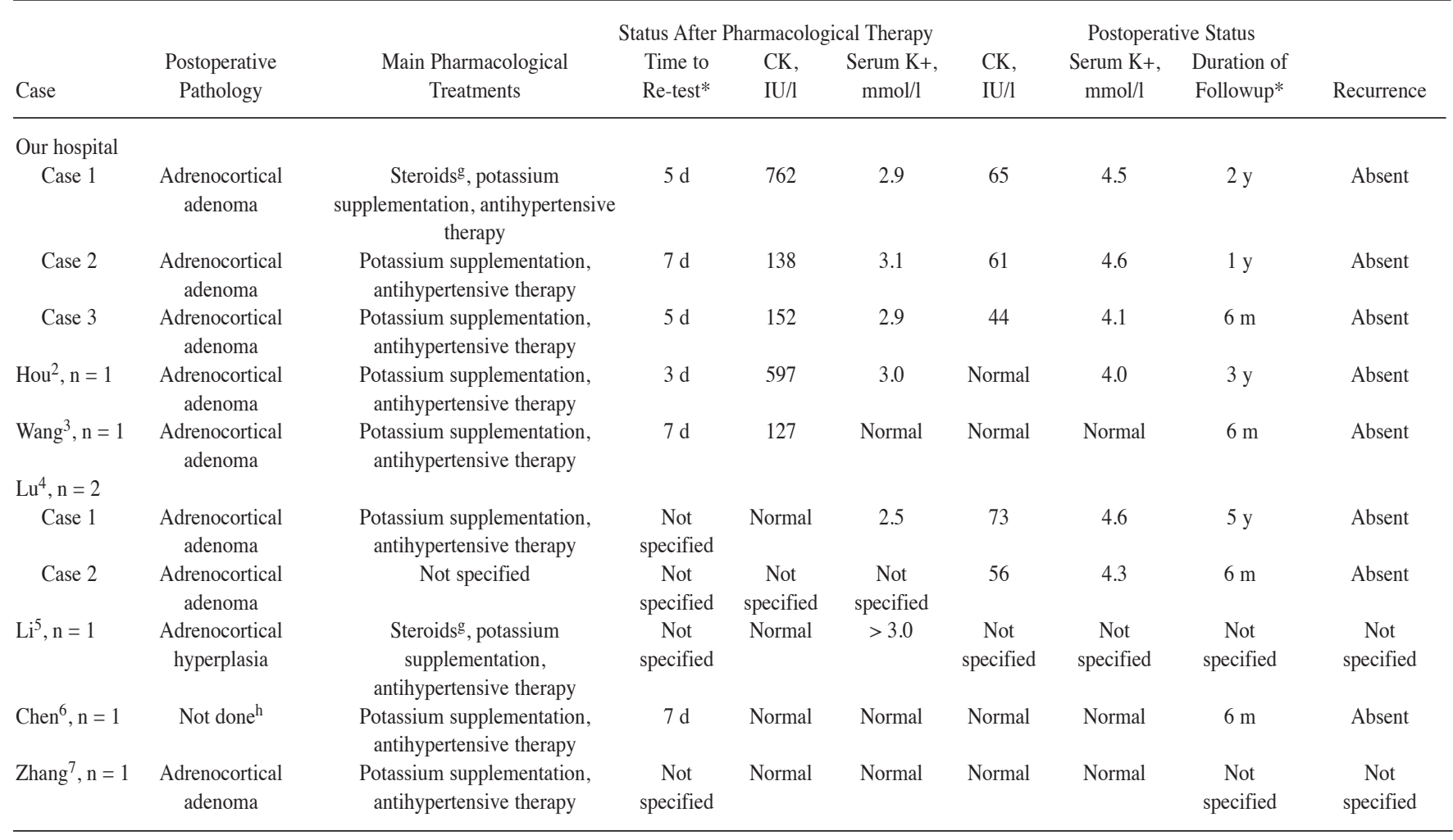

* d: days; m: months; y: years. ${ }^{a}$ History of polydipsia and increased nocturia 6 months to 3 years prior to presentation; ${ }^{b}$ limb numbness at presentation; ${ }^{c}$ history of hypokalemia 2 years prior to presentation; ${ }^{\mathrm{d}}$ supine position; ${ }^{\mathrm{e}}$ normal cortisol levels; ${ }^{\mathrm{f}}$ myoglobinuria by routine urinalysis; ${ }^{\mathrm{g}}$ short-term use of methylprednisolone $80-120$ $\mathrm{mg} /$ day; ${ }^{\mathrm{h}}$ ablative therapy of adrenal tumors was performed. NS: not specified; Myo: myogenic damage; No: no abnormalities. CK: creatine phosphokinase; CK-MB: creatine kinase isoenzyme; ALT: alanine aminotransferase; AST: aspartate aminotransferase; LDH: lactate dehydrogenase; PRA: plasma rennin activity.

longterm treatment to become alleviated and stabilized. PA prominently characterized by HM is often misdiagnosed as PM in clinical practice because patients with PA who have severe skeletal muscle injury and significantly increased muscle enzyme levels are very rarely encountered by clinicians.

Skeletal muscles are enriched with CK. Myocyte injury or increased membrane permeability due to various causes may lead to release of CK from myocytes into the blood, resulting in increased serum CK level. The level of serum CK may, to some extent, reflect the degree of involvement of muscle fibers. However, for a long time, increase in muscle enzymes has been regarded as an important clinical feature of PM. Measurement of serum muscle enzyme activity is significant for differential diagnosis of PM and determination of disease activity. Patients with PA who initially or mainly present with myalgia, muscle weakness, and increased muscle enzymes are more likely to be misdiagnosed as having PM. In this series of 10 cases, 9 were misdiagnosed with or suspected of having PM at initial diagnosis. Two patients received high doses of steroids $(80-120 \mathrm{mg} /$ day methylprednisolone) for suspected PM as evidenced by significant myalgia, muscle weakness, very high muscle enzyme levels (CK $>8000$ $\mathrm{mmol} / \mathrm{l}$ ), and myogenic damage on EMG on admission; moreover, they received aggressive potassium supplementation (4-6 g/day potassium chloride) and antihypertensive therapy for concomitant hypokalemia and hypertension. Most of the other 8 patients received only potassium supplementation and antihypertensive therapy. They experienced significant improvement in myalgia and muscle weakness 3-7 days after treatment and had considerably decreased CK level and significantly elevated serum potassium level on reexamination. The treatment course of these patients was judged to be not in line with that of PM although 3 of the patients had inflammatory cell infiltration in the muscle biopsy. Additionally, in view of the presence of concomitant hypertension, hypokalemia, and alkalemia as well as a history of hypertension and polydipsia/polyuria, these patients were considered to be more likely to have PA than PM. Further evaluations to support the diagnosis of PA were performed and surgical resection of adrenal tumors was done. Finally, the diagnosis of PA was confirmed. After surgical removal or ablation of adrenal tumors, all the patients achieved good outcomes, including rapid return of serum potassium and muscle enzymes to normal, resolution in myalgia and muscle weakness, and return of BP to normal levels. In 8 patients, who were followed for 6 months to 5 years, no recurrences were reported. This case series had many features of classic PM that met (or almost met) the classification criteria for $\mathrm{PM}^{1}$, as shown by the acute onsets of proximal muscle pain and weakness, elevated muscle enzymes, and characteristic findings on EMG and muscle biopsy. However, they had concomitant severe hypokalemia and hypertension. Their muscle enzymes decreased rapidly and muscle strength improved significantly only when potassium supplementation and antihypertensive therapy were used (with short-period use or no use of steroids) and returned rapidly to normal after surgical resection of adenomas. Their immunologic tests were normal. In addition, they did not experience any recurrence during followup. Therefore, muscle injury seen in these patients was considered to be secondary to PA. The possibility of PM or concomitant PM could then be excluded.

Muscle damage seen in patients with PA is closely associated with hypokalemia and the association has been confirmed by enzymatic and histological examinations. When serum potassium level is decreased to below $2.0 \mathrm{mEq} / 1$, a patient with PA may have marked elevation of serum muscle enzymes. In such a case, histological findings include diffuse necrosis and vacuolization of muscle fibers in damaged muscle under light microscopy, and complete dissolution of myofilaments with disappearance of sar- 
coplasmic reticulum and T-tubules in the necrotic muscle fibers under electron microscopy ${ }^{8}$. Therefore, PA-induced muscle injury is directly related to hypokalemia and falls into the category of HM because it shares similar clinical and histological features with HM. This has also been confirmed in the literature ${ }^{9,10}$. It has been known that HM is suggested to be related to hypokalemia, which is histologically mainly characterized by muscle fiber necrosis and clinically characterized by muscle pain, tenderness and weakness, elevated muscle enzymes, and typical myopathic changes on EMG. There are reports of HM due to gastrointestinal potassium loss, diarrhea, diuretic use, clay ingestion, chronic alcoholism, and Bartter syndrome ${ }^{8,9}$. PA is another cause for the development of HM. Myoglobinemia and myoglobinuria have been found in about $50 \%$ of patients with dermatomyositis and $\mathrm{PM}$, but also occurs in $\mathrm{HM}^{11,12}$. In line with previous findings, 1 patient in our series had myoglobinuria. The histopathological findings of HM usually include not only muscle fiber necrosis and vacuolar changes but also inflammatory cell infiltration and regeneration ${ }^{11}$. It is hard to distinguish HM from PM histopathologically because of similar histopathologic features. Vacuolar changes, which are rarely seen in PM, bear importance in differential diagnosis of these 2 conditions. However, in our series, vacuolar changes were not observed in 3 patients who underwent muscle biopsy. Thus HM and PM share similar clinical and histological characteristics and cannot be differentiated by determination of muscle enzymes, myoglobins, or EMG findings, or by histopathological evaluation alone. Even when vacuolar changes are observed, other myopathic disorders that may cause vacuolar changes should be excluded, such as hypokalemic periodic paralysis, inclusion body myositis, reducing body myopathy, hypothyroid myopathy, and some distal myopathies ${ }^{9,13,14}$. Nevertheless, there are significant differences between HM and PM: HM may be accompanied by profound hypokalemia, while PM is not associated with blood potassium levels; patients with HM may achieve rapid and complete improvement after correction of serum potassium to normal levels and etiology-specific interventions, and are far less likely to experience recurrence, whereas patients with PM may require longterm use of steroids to become stable and are more prone to recurrence. In our series, patients made a rapid recovery after potassium supplementation and resection of aldosterone-producing tumors and reported no recurrence during followup. The clinical course was consistent with HM rather than PM. Therefore, in the differential diagnosis of the 2 conditions, information on medical history, clinical and pathological features, and changes in clinical and laboratory manifestations after receiving potassium supplementation should be analyzed to make an accurate diagnosis.

HM and PM are both established causes of myopathy that may be easily confused because of similar presentations. Hypokalemia should be considered in the specific diagnosis and differential diagnosis of both myopathies. Special care should be taken in the diagnosis of PM. When typical signs of $\mathrm{PM}$ and profound hypokalemia are concurrently present, HM should be considered. Concomitant hypertension strongly suggests PA-induced HM. In such cases, further evidence of PA should be investigated for an accurate diagnosis and an appropriate treatment plan to prevent poor outcomes.
YAN-CHUN TANG, MD; SHAO-KUN WANG, MD; WEI-LING YUAN, MD, Department of Rheumatology, Yantai Yuhuangding Hospital, Shandong 264000, China. Address correspondence to Dr. Tang; E-mail: yt.lyls@163.com

\section{REFERENCES}

1. Bohan A, Peter JB. Polymyositis and dermatomyositis (first of 2 parts). N Engl J Med 1975;292:344-7.

2. Hou Q, Wang Y, Pei J, Ni GB. One case of primary aldosteronism complicated with severe skeletal muscle injury. Chinese J Intern Med 2004;43:144.

3. Wang D, Lao LF, Huang JQ. Primary aldosteronism complicated with elevated muscle enzymes. Clin Misdiagnosis Mistherapy 2007;20:82-3.

4. Lu YH. Two cases of primary aldosteronism simulating primary polymyositis. Chinese J Rheumatol 2005;9:635-6.

5. Li M, Zhao JM, Liu FX, Ma JJ. One case of primary aldosteronism simulating polymyositis. Chinese J Postgrad Med 2006;29:77.

6. Chen XP, Li Q, Li KH, Gao M, Huang DJ, Yin QX. Increased cardiac muscle enzymes induced by primary aldosteronism: a case report. J Clin Cardiol 2003;19:185.

7. Zhang RF, Sun XL, Wen Q. Primary aldosteronism simulating polymyositis. Clin Misdiagnosis Mistherapy 2010;23:74-5.

8. Ishikawa S, Saito T, Okada K, Atsumi T, Kuzuya T. Hypokalemic myopathy associated with primary aldosteronism and glycyrrhizine-induced pseudoaldosteronism. Endocrinol Jpn 1985;32:793-802.

9. Kasifoglu T, Korkmaz C, Pasaoglu O. Conn's syndrome (primary aldosteronism) simulating polymyositis. Rheumatol Int 2005;25:133-4.

10. Chow CP, Symonds CJ, Zochodne DW. Hyperglycaemia, lumbar plexopathy and hypokalemic rhabdomyolysis complicating Conn's syndrome. Can J Neurol Sci 1997;24:67-9.

11. Becker NJ, Hinman M, Giles MN, Kepes JJ, Abdou NI. Polymyositis with hypokalemia: correction with potassium replacement in the absence of steroids. J Rheumatol 1987;14:1042-4.

12. Mijares RP. Hypokalemic rhabdomyolysis secondary to pseudohyperaldosteronism due to the use of a lotion containing 9-alphafluoropredisolone. Nephron 1986;43:232-3.

13. Jongen PJ, Ter Laak HJ, Stadhouders AM. Rimmed basophilic vacuoles and filamentous inclusion in neuromuscular disorders. Neuromuscul Disord 1995;5:31-8.

14. Dai L, Zheng DH, Mo YQ, Wu YM, Zhang BY. Retrospective analysis of fifty-seven cases of polymyositis-like syndrome in hypothyroidism patients. Chinese J Rheumatol 2007;11:738-41.

J Rheumatol 2011;38:7; doi:10.3899/jrheum.110034 